\title{
Suppression of mitochondrial respiration with local anesthetic ropivacaine targets breast cancer cells
}

\author{
Xiaodan Gong ${ }^{1 \#}$, Jiapeng Dan ${ }^{2 \#}$, Fang $\mathrm{Li}^{2}$, Lishen Wang ${ }^{2}$ \\ ${ }^{1}$ Department of Anesthesiology, The Second Clinical Medical College, Yangtze University, Jingzhou 434020, China; ${ }^{2}$ Department of Anesthesiology, \\ Jingzhou Central Hospital, Jingzhou 434020, China \\ Contributions: (I) Conception and design: X Gong, L Wang; (II) Administrative support: F Li; (III) Provision of study materials or patients: X Gong, \\ J Dan, F Li; (IV) Collection and assembly of data: X Gong, J Dan, L Wang; (V) Data analysis and interpretation: X Gong, J Dan, L Wang; (VI) \\ Manuscript writing: All authors; (VII) Final approval of manuscript: All authors. \\ "These authors contributed equally to this work. \\ Correspondence to: Lishen Wang. Department of Anesthesiology, Jingzhou Central Hospital, Renmin Road 1, Jingzhou 434020, China. \\ Email: gxd8015363@163.com.
}

Background: Retrospective studies on cancer patients who have received local anesthesia show a favorable decrease in tumor metastasis and recurrence. However, the mechanisms underlying the benefits of local anesthesia on cancer recurrence are not well understood.

Methods: In this study, we investigated the biological effects of ropivacaine on breast cancer cells and the mechanisms of its action with emphasis on mitochondrial respiration.

Results: Ropivacaine significantly inhibited growth, survival, and anchorage-independent colony formation in two human breast cancer cell lines. It also acted synergistically with a 5-FU in breast cancer cells. Mechanistically, ropivacaine was found to inhibit mitochondrial respiration by suppressing mitochondrial respiratory complex I and II activities, leading to energy depletion, and oxidative stress and damage. The inhibitory effects of ropivacaine in breast cancer cells were abolished in mitochondrial respiration-deficient $\rho 0$ cells, indicating that mitochondrial respiration is essential for the mechanism of action of ropivacaine. Ropivacaine inhibited phosphorylation of Akt, mTOR, rS6, and EBP1 in breast cancer cells, suggesting the association between Akt/mTOR signaling pathway and mitochondrial functions in breast cancer.

Conclusions: Our work clearly demonstrates the inhibitory effects of ropivacaine in breast cancer by disrupting mitochondrial function. Our findings provide a proper understanding of how local anesthetics reduce the risk of tumor recurrence, and thus, support the use of ropivacaine for surgery and to control pain in patients with breast cancer.

Keywords: Breast cancer; ropivacaine; mitochondrial respiration; Akt/mTOR

Submitted Dec 06, 2017. Accepted for publication Apr 25, 2018.

doi: $10.21037 /$ jtd.2018.05.21

View this article at: http://dx.doi.org/10.21037/jtd.2018.05.21

\section{Introduction}

Anesthetics are frequently used for surgical tumor removal and the management of chronic pain in cancer patients (1). In addition to their known effects on preoperative, intraoperative, and postoperative pain management, there is emerging evidence that specific anesthetics have an anticancer effect (2-4). Several retrospective studies suggest that local anesthesia reduces tumor metastasis and recurrence in patients with breast, prostate, or colon cancer undergoing mastectomy, prostatectomy, or surgery, respectively $(5,6)$. Interestingly, many general anesthetics (e.g., opioids) are immunosuppressive and decrease a patient's immune defenses against malignant progression (7). In contrast, some local anesthetics have an inhibitory role in tumor growth and invasion (8). 
Amide-linked local anesthetics, such as ropivacaine, are commonly used for thoracic paravertebral block in breast cancer surgery (9) and to reduce chronic pain (10). The mechanisms of action of anesthetics are due to their ability to block the voltage-gated sodium channel (10). Notably, accumulating evidence shows that amide-linked local anesthetics directly inhibit growth, migration, and survival of various cancer cell lines (4,11-13). In addition to functioning as a voltage-gated sodium-channel inhibitor, ropivacaine has been shown to inhibit mitochondrial biogenesis, increase reactive oxygen species, activate mitogen-activated protein kinase (MAPK) pathways, and decrease Src activity $(8,11)$. Although the effects of ropivacaine on cancer seem to be specific to tumor cell type, the underlying molecular mechanisms are unknown.

Therefore, in this study, we investigated the effects of ropivacaine on breast cancer using two representative human breast cancer cell lines. We examined the growth, survival, and anchorage-independent colony formation in cells after ropivacaine treatment and analyzed the underlying mechanisms. Our findings show that ropivacaine has direct inhibitory effects by disrupting mitochondrial function and suppressing Akt/mTOR pathway in breast cancer cells. Our findings also demonstrate synergy between ropivacaine and standard chemotherapeutic agents.

\section{Methods}

\section{Cell culture and generation of mitochondrial respiration- deficient $\rho 0$ cell line}

MDA-MB-468 and SkBr cell lines were obtained from American Type Culture Collection and cultured in Minimal Essential Media supplemented with 10\% heatinactivated FBS and $2 \mathrm{mM} \mathrm{L}$-glutamine (Invitrogen, USA). Mitochondria DNA-deficient $\mathrm{SkBr} \rho 0$ cell line was established according to the method described by King and Attardi (14). Briefly, cells were cultured as described above and selected with $1 \mu \mathrm{g} / \mathrm{mL}$ ethidium bromide (EtBr; Sigma, USA), supplemented with $200 \mu \mathrm{M}$ uridine and $1 \mathrm{mM}$ sodium pyruvate (Sigma, MO, USA) for 40 days, and thereafter maintained in the above media without $\mathrm{EtBr}$.

\section{Measurement of proliferation and apoptosis}

Cells were treated with ropivacaine (Sigma, USA) and 5 -fluorouracil (5-FU, Sigma, USA) alone or in combination for 3 days. Cell proliferation and apoptosis was determined by the CellTiter 96R AQueous One Solution Cell Proliferation assay kit (Promega, USA) and flow cytometry with Annexin V/7-AAD (Beckman Coulter, USA), respectively, using the same protocol as reported in our previous study (15).

\section{Soft agar colony formation assay}

Soft agar colony formation was carried out using CytoSelect 96-Well Cell Transformation Assay (Cell Biolabs Inc, USA) according the manufacturer's protocol. Briefly, a bottom layer of $1.2 \%$ agar solution was plated and solidified. A top layer of equal volumes of $1.2 \%$ agar solution, culture medium, and cell suspension (1,000 cells/well) was replated. Culture medium $(100 \mu \mathrm{L})$ was added to the top layer of the soft agar and replaced with fresh medium every 3 days. The cells were incubated for $6-8$ days at $37^{\circ} \mathrm{C}$ and $5 \% \mathrm{CO}_{2}$. Colonies were stained with $0.4 \%$ crystal violet (Sigma, USA) and counted under a light microscope.

\section{Measurement of oxygen consumption rate (OCR), mitochondrial membrane potential, and ATP level}

Cells were treated with ropivacaine for $24 \mathrm{~h}$ prior to measuring OCR, membrane potential, and ATP. Cells were then equilibrated to the un-buffered medium in a $\mathrm{CO}_{2}$-free incubator and transferred to the Seahorse XF24 extracellular flux analyzer (Seahorse Bioscience, USA). Analyses were performed at basal condition. Three mitochondrial inhibitors, oligomycin $(1 \mathrm{mM})$, carbonyl cyanide-p-trifluoromethoxyphenylhydrazone (FCCP; $300 \mathrm{mM})$, and rotenone $(1 \mathrm{mM})$, were sequentially injected for measuring OCR under maximal condition. All injection reagents were adjusted to $\mathrm{pH} 7.4$ on the day of the assay. The Seahorse XF-24 software calculated OCR automatically. Mitochondrial membrane potential was determined by flow cytometry with 5,5',6,6'-tetrachloro1,1',3,3'-tetraethyl benzimidazolylcarbocyanine iodide (JC1, Invitrogen) staining according to the manufacturer's protocol. ATP levels were measured by CellTiter-Glo Luminiescent Cell Viability Assay (Promega, WI, USA).

\section{Measurement of oxidative stress and damage}

Cells were treated with ropivacaine for $24 \mathrm{~h}$. Mitochondrial superoxide was measured by staining cells with MitoSox Red according to the manufacturer's protocol. The absorbance at ex/em of $510 / 580 \mathrm{~nm}$ was measured using Spectramax M5 microplate reader (Molecular Devices). Oxidative DNA 
damage was determined using the OxiSelect Oxidative DNA Damage ELISA Kit (Cell Biolabs).

\section{Measurement of mitochondrial respiratory complex activity}

Cells were treated with ropivacaine for $24 \mathrm{~h}$ prior to mitochondrial respiratory complex activity measurement. The activities of mitochondrial respiratory complexes I, II, IV, and V were measured using Mitochondrial Complex I, II, IV, and V Activity Assay Kits (Novagen, USA), respectively, according to the manufacturer's protocol.

\section{Western blot (WB) analyses}

Cells were lysed using 4\% SDS containing a phosphatase inhibitor cocktail (Invitrogen). Total protein concentration was determined using the bicinchoninic acid protein assay kit (Thermo Scientific, USA). Equal amounts of total proteins were loaded and resolved using denaturing SDSPAGE, and analyzed by WB using antibodies against phosphorylated and total Akt, mTOR, 4EBP1 and S6, and $\beta$-actin (Cell technology, USA).

\section{Statistical analyses}

Statistical analyses were performed using unpaired Student's $t$-test. A P value $<0.05$ was considered statistically significant.

\section{Results}

\section{Ropivacaine inhibits breast cancer growth, survival, and colony formation}

Ropivacaine has been shown to inhibit the growth of various types of cancer cells with an effective dose range of $0.1-10 \mathrm{mM}(16,17)$. We therefore evaluated the effects of ropivacaine at concentrations of $0.1,0.5$, and $1 \mathrm{mM}$ on the growth and survival of MDA-MB-468 and SkBr human breast cancer cells. MDA-MB-468 is a triple-negative breast cancer cell line with genetic amplification of the epithelial growth factor receptor (EFGR), whereas the $\mathrm{SkBr}-3$ cell line overexpresses human epithelial growth factor receptor 2 (HER2) enriched (18). After $72 \mathrm{~h}$ of treatment, ropivacaine at concentrations of 0.5 and $1 \mathrm{mM}$ significantly inhibited proliferation and induced apoptosis in a concentrationdependent manner in MDA-MB-468 and SkBr-3 cells (Figure 1A,B). SkBr-3 cells appear to be more sensitive to ropivacaine than MDA-MB-468 cells.

Anchorage-independent colony formation assay has been demonstrated to specifically assess the propagation and differentiation of subpopulations with "stem cell" properties within a cell line (19). We found that ropivacaine significantly inhibited anchorage-independent colony formation of MDA-MB-468 and SkBr-3 cells (Figure 1C,D). Interestingly, ropivacaine at $0.5 \mathrm{mM}$ significantly inhibits colony formation but does not affect growth and survival, suggesting that the subpopulations of breast cancer cells with "stem cell" properties are more sensitive to ropivacaine than the bulky cell lines.

\section{Ropivacaine disrupts mitochondrial function and induces oxidative stress in breast cancer cells}

The inhibitory effects of ropivacaine on mitochondrial functions have been observed in various cell types $(20,21)$. To investigate whether the effects of ropivacaine on breast cancer cells are due to mitochondrial dysfunction, we first examined mitochondrial membrane potential and mitochondrial respiration in cells exposed to ropivacaine. Ropivacaine did not affect mitochondrial membrane potential in MDA-MB-468 and $\mathrm{SkBr}-3$ cells (Figure $2 A$ ). However, breast cancer cells treated with ropivacaine had reduced baseline and maximal mitochondrial respiration as measured by Seahorse XF24 extracellular flux analyzer (Figure 2B,C), suggesting that ropivacaine inhibits mitochondrial respiration. We further demonstrated that ropivacaine specifically suppressed mitochondrial respiratory complexes I and II, but did not suppress complexes IV and V in breast cancer cells (Figure 2D). This suggests that ropivacaine inhibits mitochondrial respiration by disrupting complex I and II activities. Consistent with the inhibition of mitochondrial respiration, a significant reduction in ATP levels and accumulation of oxidative stress and damage as shown by the increased levels of mitochondrial superoxide and $8-\mathrm{OHdG} / \mathrm{mg}$ DNA were observed in ropivacainetreated breast cancer cells (Figure 2E,F,G). These results demonstrate that ropivacaine disrupts mitochondrial functions, which in turn leads to energy depletion, oxidative stress, and damage in breast cancer.

\section{The inbibitory effects of ropivacaine are abolished in mitochondrial respiration-deficient breast cancer $\rho 0$ cells}

By culturing cells in the presence of ethidium bromide, we 
A

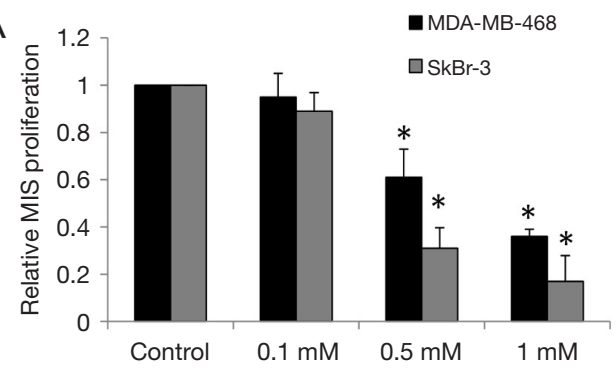

C
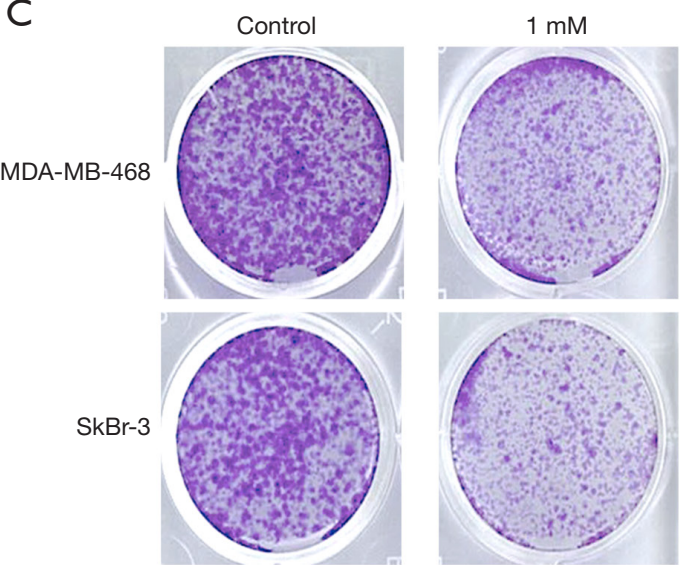

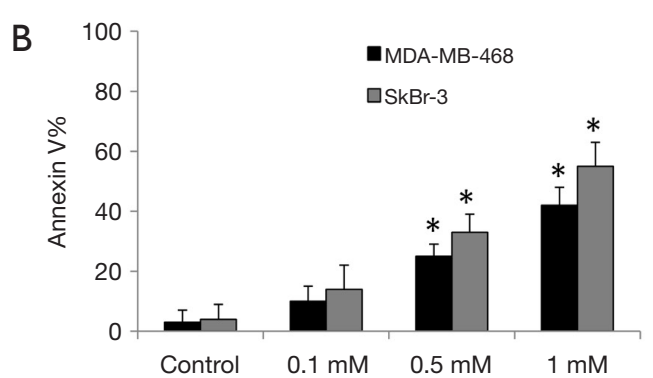

D

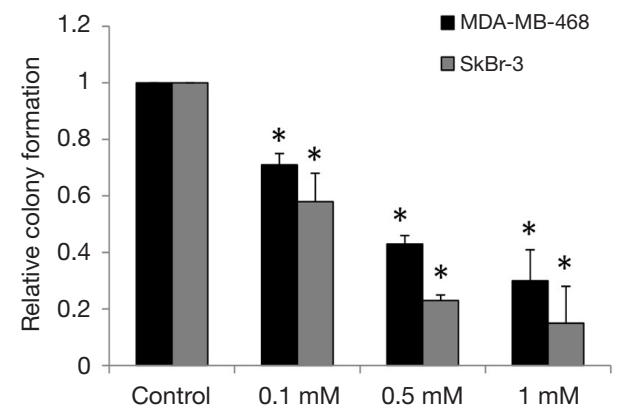

Figure 1 Ropivacaine targets multiple aspects of breast cancer cells. Ropivacaine at 0.5 and $1 \mathrm{mM}$ significantly decreases proliferation (A) and increases Annexin V (B) of MDA-MB-468 and SkBr cells. Annexin V-positive cells were considered as apoptotic cells. Representative photos taking at 10 days of an anchorage-independent colony-forming assay (C) and quantification of colonies (colonies were stained with $0.4 \%$ crystal violet, followed by gentle PBS wash. Magnification was taken at 50x) (D) showing the inhibitory effect of ropivacaine on colony formation of breast cancer cells. The data were derived from three independent experiments and presented as mean $\pm \mathrm{SEM}$. * $\mathrm{P}<0.05$, compared to control.

established $\rho 0$ cells that lack mitochondrial DNA and thus are incapable of performing mitochondrial respiration (14). Although the generation of $\rho 0$ cells from $\mathrm{SkBr}-3$ cells was unsuccessful, we established $\rho 0$ cells from MDAMB-468 which have a minimal level of baseline oxygen consumption rate and are non-responsive to uncoupling of mitochondrial oxidative phosphorylation via FCCP (Figure $3 A$ ). We further observed a significantly decreased basal level of ATP in breast cancer $\rho 0$ cells which is likely due to their mitochondrial respiration defects (Figure 3B). Notably, the inhibitory effects of ropivacaine on cellular ATP levels, mitochondrial superoxide, 8-OHdG/ mg DNA, and Annexin $\mathrm{V}$ were observed in parental MDA-MB-468 but not in MDA-MB-468 $\rho 0$ cells (Figure $3 B, C, D, E)$. Taken together, mitochondrial functions are essential for the inhibitory effects of ropivacaine on breast cancer cells.

\section{Ropivacaine significantly enhances 5-FU's effects in breast cancer cells by suppressing Akt/mTOR signaling patbway}

To investigate whether ropivacaine acts synergistically with a standard chemotherapeutic agent in breast cancer cells, we compared the effects of the combination of ropivacaine and 5-FU with those of each drug alone. We designed and performed combination studies using the concentration of ropivacaine or 5-FU that slightly or moderately affects breast cancer cells. Our results showed that combination of ropivacaine and 5-FU was significantly more effective than ropivacaine or 5 -FU alone at inhibiting growth, survival, and colony formation (Figure 4A,B,C). Of note, nearly complete inhibition of the 
A

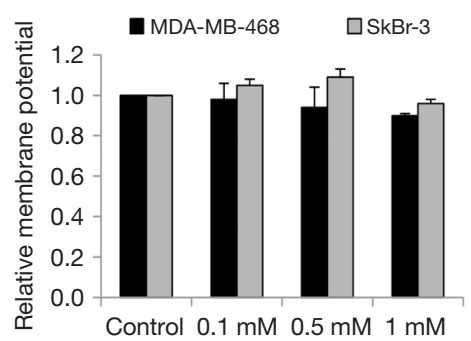

B

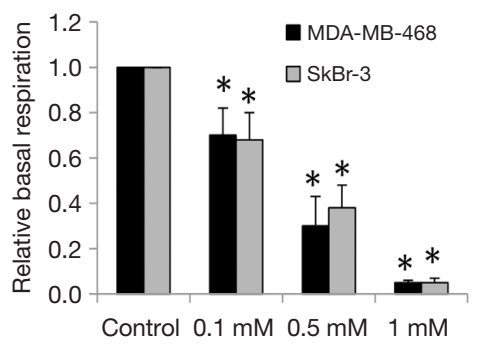

C

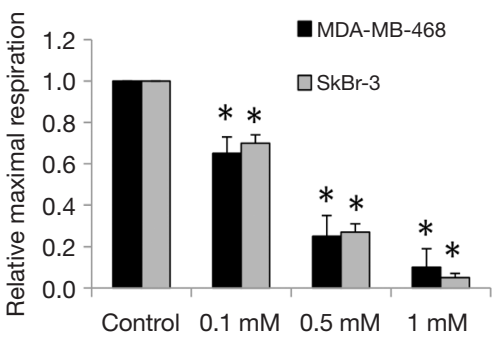

$\mathrm{D}$
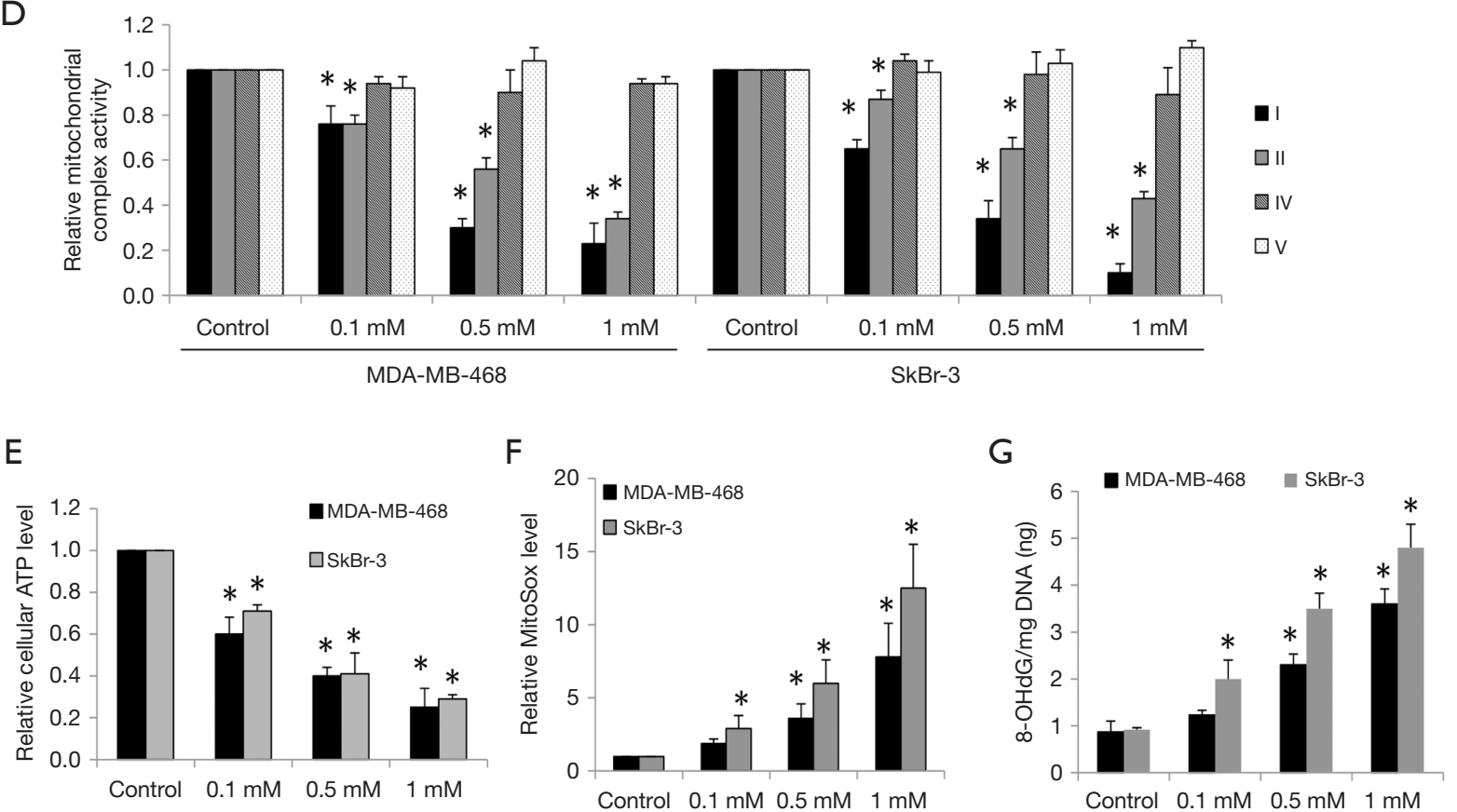

Figure 2 Ropivacaine inhibits mitochondrial respiration and induces oxidative stress in breast cancer. (A) Ropivacaine does not affect mitochondrial membrane potential. Ropivacaine dose-dependently suppresses basal (B) and maximal OCR (C). (D) Ropivacaine inhibits activities of mitochondrial complex I and II but not IV and V. Ropivacaine decreases ATP levels (E) and increases mitochondrial superoxide (F) and 8-OHdG/mg DNA levels (G) in breast cancer cells. The data were derived from three independent experiments and presented as mean $\pm \mathrm{SEM}$. *, $\mathrm{P}<0.05$, compared to control.

multiple biological activities of breast cancer was observed with the drug combination.

Because the Akt/mTOR pathway has been shown to control mitochondrial activity and biogenesis $(22,23)$, we assessed the effects of ropivacaine on the essential molecules involved in the Akt/mTOR signaling pathway. We observed decreased phosphorylation of Akt (S473) and mTOR (S2481) in MDA-MB-468 and SkBr cells exposed to ropivacaine (Figure 4D). Phosphorylation of mTOR signaling downstream effectors, such as the ribosomal S6 protein $(\mathrm{rS} 6)$ and 4EBP1, was also decreased by ropivacaine
(Figure 4D). These results suggest that ropivacaine augments the effects of chemotherapeutic agents in breast cancer cells, likely by suppressing the Akt/mTOR signaling pathway.

\section{Discussion}

Substantial evidence has shown that anesthetic management of a cancer patient could potentially influence long-term outcome. Although general anesthetics (e.g., opioids and volatile agents) have been speculated to promote tumor 
A
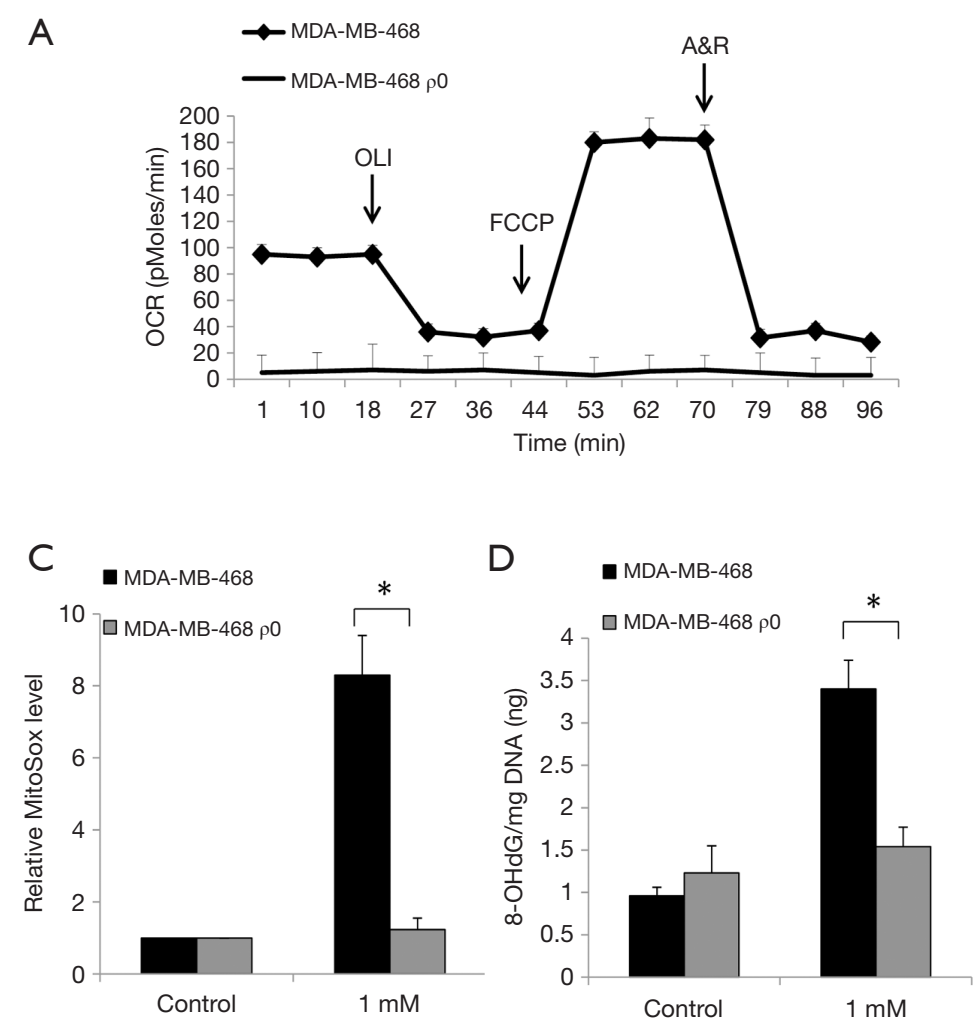
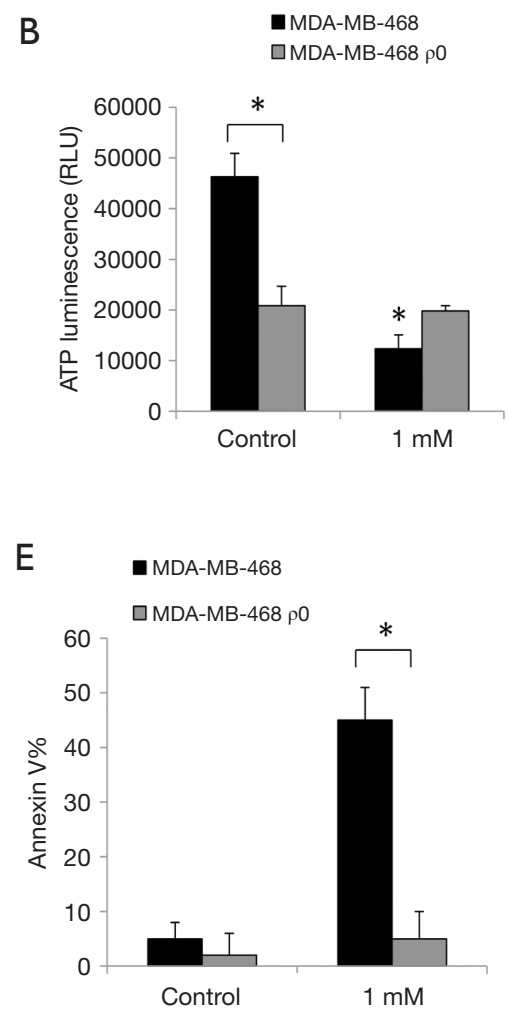

Figure 3 Breast cancer $\rho 0$ cells are resistant to ropivacaine treatment. (A) MDA-MB-468 $\rho 0$ cells have minimal basal level of OCR and are non-responsive to FCCP. (B) $\rho 0$ cells are resistant to ropivacaine-induced depletion of ATP. ATP levels are decreased in $\rho 0$ cells than parental cells. $\rho 0$ cells are resistant to ropivacaine in increasing levels of mitochondrial superoxide (C), 8-OHdG/mg DNA (D) and Annexin $\mathrm{V}(\mathrm{E})$. The data were derived from three independent experiments and presented as mean $\pm \mathrm{SEM}$. * $\mathrm{P}<0.05$, compared to MDA-MB-468.

metastasis due to their immunosuppressive and proangiogenic effects, retrospective clinical trials on cancer patients suggest that regional and local anesthesia are independent beneficial factors for tumor recurrence after cancer surgery $(4,24)$. Preclinical data also suggest that many local anesthetics have anti-proliferation and proapoptotic activities on cancer cell cultures and xenograft mouse model $(12,17,25)$. Better understanding of the potential role of anesthetics in cancer will be helpful to select the optimal anesthetic regimens for better treatment outcomes in cancer patients. The aim of this study was to investigate the effects of clinically used local anesthetics on breast cancer cell proliferation, colony formation, and survival.

The human breast cancer cell lines we selected to demonstrate the potential effects of ropivacaine were MDAMB-468 and SkBr-3 cells. These two cell lines represent different cellular origin and display genetic heterogeneity with HER2 or EFGR overexpression which are highly resistant to standard chemotherapy (18). We show that ropivacaine inhibits proliferation, colony formation, and survival of MDA-MB-468 and SkBr-3 cells (Figure 1). Of note, ropivacaine seems to preferentially target breast cancer subpopulations with stem cell properties rather than bulky cells. The inhibitory effects of ropivacaine on proliferation, survival, and invasion have been demonstrated in hepatocellular carcinoma and lung and colon cancer $(11,16,26)$. In line with these studies, our findings add breast cancer to the growing list of cancers inhibited by ropivacaine. We further show that the combination of ropivacaine and 5-FU (a standard chemotherapeutic agent for breast cancer treatment) achieves almost complete inhibition of all aspects of breast cancer activities when using a concentration of 5-FU that marginally inhibits breast cancer cells as single drug alone (Figure $4 A, B, C$ ). This suggests that ropivacaine is a useful addition to the treatment armamentarium for breast cancer. We and others have consistently demonstrated the direct anti- 

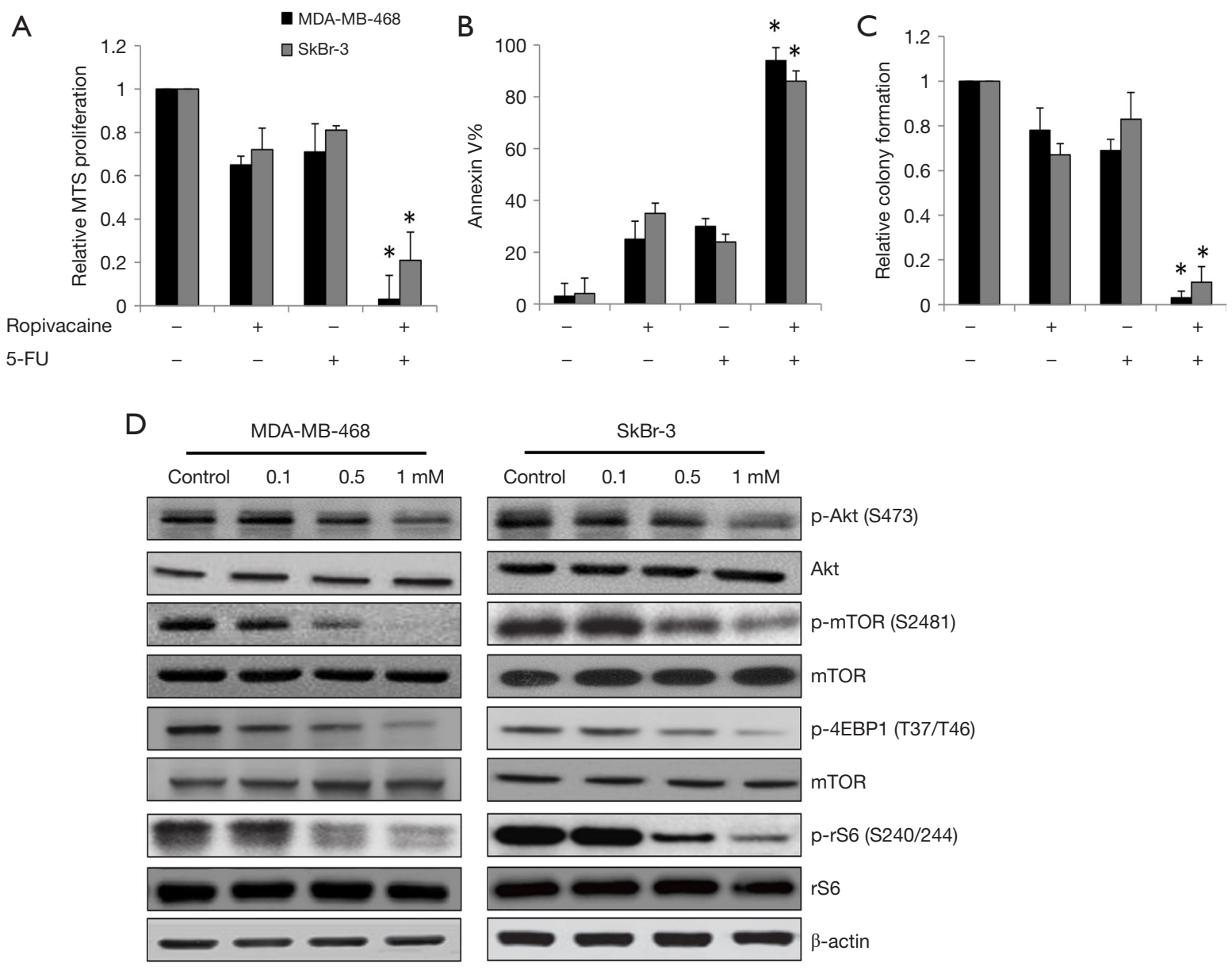

Figure 4 Ropivacaine augments 5-FU's inhibitory effects through suppresses mTOR signaling pathway in breast cancer cells. Ropivacaine significantly augments the effects of 5-FU in decreasing proliferation (A), inducing apoptosis (B) and inhibiting colony formation (C) in MDA-MB-468 and SkBr cells. $0.5 \mathrm{mM}$ of ropivacaine and $1 \mu \mathrm{M}$ of 5 -FU were used for the combination study. *, $\mathrm{P}<0.05$, compared to ropivacaine or 5-FU alone. (D) The phosphorylation of Akt, mTOR, rS6 and 4EBP1 are decreased by ropivacaine in breast cancer cells.

cancer effect of ropivacaine in cell-based cancer models. Other amide-linked local anesthetics, such as lidocaine and bupivacaine, have similar effects on cancer $(27,28)$. Because all of the current studies are cell culture systembased, further investigations are warranted to evaluate the efficacy of amide-linked local anesthetics in patient-derived xenograft mouse models predictive of clinical outcomes, and ultimately clinical trials.

Although the inhibitory effects of ropivacaine have been reported in various cancers, the underlying mechanisms are not well understood. We demonstrated that mitochondrial dysfunction is the mechanism of action of ropivacaine in breast cancer. Ropivacaine significantly decreases mitochondrial respiration without affecting membrane potential by targeting mitochondrial respiratory complexes
I and II but not IV or V (Figure $2 A, B, C, D$ ). The loss of activities in mitochondrial respiration-deficient cells confirms mitochondrial respiration as the essential target of ropivacaine in breast cancer (Figure 3). This is consistent with previous studies on the effects of ropivacaine on mitochondrial oxidation and bioenergetics in normal myocardial cells $(20,29)$. Our findings extend the findings of previous work by demonstrating the inhibitory effects of ropivacaine on tumor cells. mTOR directly controls mitochondrial functions and inhibition of mTOR suppresses mitochondrial respiration in cancer cells (23). In agreement with this, we observed the decreased phosphorylation levels of essential molecules involved in Akt/mTOR signaling pathway in breast cancer cells exposed to ropivacaine (Figure 4D). Our work and that of others suggest that 
inhibition of Akt/mTOR negatively affects mitochondrial function and subsequently induces oxidative stress in breast cancer cells.

In summary, we are the first to systematically demonstrate the inhibitory effects of ropivacaine on breast cancer cells and to show mitochondrial dysfunction as its underlying mechanism. The synergism between ropivacaine and 5'FU suggests that ropivacaine could be a useful addition to breast cancer treatment. Our study provides the fundamental evidence for clinical observations on the beneficial role of local anesthesia in reducing breast cancer recurrence. Since local anesthetics, which are usually given using an indwelling catheter, are also used for postoperative pain control, it is worthy of investigating whether continuous administration of local anesthetics by indwelling catheter has better efficacy than single administration during surgery. It is also noteworthy to investigate the various indwelling catheter locations, such as the surgical wound, intra-articular, or intrapleural area, to obtain the maximum benefit with minimal side effects using low drug concentrations.

\section{Acknowledgements}

Funding: This work was supported by a research grant provided by Jingzhou Central Hospital (201406016).

\section{Footnote}

Conflicts of Interest: The authors have no conflicts of interest to declare.

\section{References}

1. Gudaityte J, Dvylys D, Simeliunaite I. Anaesthetic challenges in cancer patients: current therapies and pain management. Acta Med Litu 2017;24:121-7.

2. Snyder GL, Greenberg S. Effect of anaesthetic technique and other perioperative factors on cancer recurrence. $\mathrm{Br} \mathrm{J}$ Anaesth 2010;105:106-15.

3. Tavare AN, Perry NJ, Benzonana LL, et al. Cancer recurrence after surgery: direct and indirect effects of anesthetic agents. Int J Cancer 2012;130:1237-50.

4. Mao L, Lin S, Lin J. The effects of anesthetics on tumor progression. Int J Physiol Pathophysiol Pharmacol 2013;5:1-10.

5. Exadaktylos AK, Buggy DJ, Moriarty DC, et al. Can anesthetic technique for primary breast cancer surgery affect recurrence or metastasis? Anesthesiology 2006;105:660-4.

6. Biki B, Mascha E, Moriarty DC, et al. Anesthetic technique for radical prostatectomy surgery affects cancer recurrence: a retrospective analysis. Anesthesiology 2008;109:180-7.

7. Plein LM, Rittner HL. Opioids and the immune system friend or foe. Br J Pharmacol 2017. [Epub ahead of print].

8. Piegeler T, Votta-Velis EG, Liu G, et al. Antimetastatic potential of amide-linked local anesthetics: inhibition of lung adenocarcinoma cell migration and inflammatory Src signaling independent of sodium channel blockade. Anesthesiology 2012;117:548-59.

9. Sahu A, Kumar R, Hussain M, et al. Comparisons of single-injection thoracic paravertebral block with ropivacaine and bupivacaine in breast cancer surgery: A prospective, randomized, double-blinded study. Anesth Essays Res 2016;10:655-60.

10. Heavner JE. Local anesthetics. Curr Opin Anaesthesiol 2007;20:336-42.

11. Wang HW, Wang LY, Jiang L, et al. Amide-linked local anesthetics induce apoptosis in human non-small cell lung cancer. J Thorac Dis 2016;8:2748-57.

12. Chang YC, Hsu YC, Liu CL, et al. Local anesthetics induce apoptosis in human thyroid cancer cells through the mitogen-activated protein kinase pathway. PLoS One 2014;9:e89563.

13. Kobayashi K, Ohno S, Uchida S, et al. Cytotoxicity and type of cell death induced by local anesthetics in human oral normal and tumor cells. Anticancer Res 2012;32:2925-33.

14. Hashiguchi K, Zhang-Akiyama QM. Establishment of human cell lines lacking mitochondrial DNA. Methods Mol Biol 2009;554:383-91.

15. Yu M, Li R, Zhang J. Repositioning of antibiotic levofloxacin as a mitochondrial biogenesis inhibitor to target breast cancer. Biochem Biophys Res Commun 2016;471:639-45.

16. Le Gac G, Angenard G, Clement B, et al. Local Anesthetics Inhibit the Growth of Human Hepatocellular Carcinoma Cells. Anesth Analg 2017;125:1600-9.

17. Lucchinetti E, Awad AE, Rahman M, et al. Antiproliferative effects of local anesthetics on mesenchymal stem cells: potential implications for tumor spreading and wound healing. Anesthesiology 2012;116:841-56.

18. Sorlie T, Perou CM, Tibshirani R, et al. Gene expression patterns of breast carcinomas distinguish tumor subclasses 
with clinical implications. Proc Natl Acad Sci U S A 2001;98:10869-74.

19. Gao CF, Xie Q, Su YL, et al. Proliferation and invasion: plasticity in tumor cells. Proc Natl Acad Sci U S A 2005;102:10528-33.

20. Zhang S, Yao S, Li Q. Effects of ropivacaine and bupivacaine on rabbit myocardial energetic metabolism and mitochondria oxidation. J Huazhong Univ Sci Technolog Med Sci 2003;23:178-9, 183.

21. Grishko V, Xu M, Wilson G, et al. Apoptosis and mitochondrial dysfunction in human chondrocytes following exposure to lidocaine, bupivacaine, and ropivacaine. J Bone Joint Surg Am 2010;92:609-18.

22. Morita M, Gravel SP, Chenard V, et al. mTORC1 controls mitochondrial activity and biogenesis through 4E-BP-dependent translational regulation. Cell Metab 2013;18:698-711.

23. Ramanathan A, Schreiber SL. Direct control of mitochondrial function by mTOR. Proc Natl Acad Sci U S A 2009;106:22229-32.

24. Gottschalk A, Sharma S, Ford J, et al. Review article: the role of the perioperative period in recurrence after cancer surgery. Anesth Analg 2010;110:1636-43.

25. Yoon JR, Whipple RA, Balzer EM, et al. Local anesthetics inhibit kinesin motility and microtentacle protrusions in human epithelial and breast tumor cells. Breast Cancer Res Treat 2011;129:691-701.

26. Baptista-Hon DT, Robertson FM, Robertson GB, et al. Potent inhibition by ropivacaine of metastatic colon cancer SW620 cell invasion and NaV1.5 channel function. Br J Anaesth 2014;113 Suppl 1:i39-i48.

27. Zhang L, Hu R, Cheng Y, et al. Lidocaine inhibits the proliferation of lung cancer by regulating the expression of GOLT1A. Cell Prolif 2017;50.

28. Xuan W, Zhao H, Hankin J, et al. Local anesthetic bupivacaine induced ovarian and prostate cancer apoptotic cell death and underlying mechanisms in vitro. Sci Rep 2016;6:26277.

29. Sztark F, Malgat M, Dabadie P, et al. Comparison of the effects of bupivacaine and ropivacaine on heart cell mitochondrial bioenergetics. Anesthesiology 1998;88:1340-9.
Cite this article as: Gong X, Dan J, Li F, Wang L. Suppression of mitochondrial respiration with local anesthetic ropivacaine targets breast cancer cells. J Thorac Dis 2018;10(5):2804-2812. doi: 10.21037/jtd.2018.05.21 